\title{
ADULT-ONSET STILL'S DISEASE WITH PULMONARY ARTERIAL HYPERTENSION
}

\author{
Samila Costa Pinheiro Guerra Barros ${ }^{1, *}$, Dawton Yukito Torigoe ${ }^{1}$, Luiz Felipe Adsuara de Sousa ${ }^{1}$, Renata Sanzovo Pires de
} Campos Bellan ${ }^{1}$, Ana Carolina Quatrini Bortolli ${ }^{1}$

1. Faculdade de Ciências Médicas da Santa Casa de São Paulo, São Paulo (SP), Brazil.

*Corresponding author: samilacpg@hotmail.com

\section{BACKGROUND}

The most common cardiopulmonary manifestations in adult-onset Still's disease (AOSD) are transient pneumonitis, pleuritis, pericarditis, pericardial tamponade and myocarditis. Pulmonary arterial hypertension (PAH) is a rare complication associated with significant mortality. A case of AOSD with manifestation of PAH is described.

\section{CASE REPORT}

Male, 64 years old, started in 2015, a progressive weight loss, diffuse intermittent cutaneous lesions in chest and upper limbs, associated with daily fever, mainly at night, fatigue, arthralgia in shoulders and knees, arthritis in wrists, odynophagia and mild dyspnea. Investigation showed lymphadenopathy (cervical, axillary, thoracic and inguinal). Biopsies of the cervical and inguinal lymph node showed reactive hyperplasia excluding neoplastic and infectious diseases. In 2019, he was admitted to our service, presenting hyperemic punctate lesions in limbs, hepatomegaly, wrist synovitis and cervical and inguinal lymphadenopathy. Laboratory tests showed leukocytosis, elevated inflammatory tests, absence of renal and hepatic changes, autoantibodies and markers of neoplasia screening. Echocardiogram was normal. Computed tomography (CT) of the chest with slight ground-glass opacity and abdomen with hepatosplenomegaly. The patient met Yamaguchi's criteria for AOSD and prednisone was started. After 2 months, symptoms improved, with the exception of dyspnea, when methotrexate was introduced. Subsequently, an increased dose of methotrexate with taper off corticosteroids had a good clinical and laboratory response but dyspnea was still worsening. Investigation continued with a new chest CT that showed lymphadenopathy in several chains, maintaining discrete areas of ground glass. The new echocardiogram showed pulmonary hypertension (PAP $75 \mathrm{mmHg}$ ); spirometry a nonspecific ventilatory disorder and scintigraphy low probability of pulmonary embolism.

\section{CONCLUSION}

Cardiopulmonary manifestations in AOSD are described in $30-40 \%$ of cases, represented mainly by pleural and pericardial disorders, being less frequent than cutaneous and articular conditions. Pulmonary arterial hypertension is a very rare complication with only 12 cases reported by the year 2017. The association HAP-DAS is predominant in females, making our case even more rare due to its involvement in males. The development of PAH in AOSD occurs an average of 24 months after diagnosis. In our case, $\mathrm{PAH}$ was evidenced 48 months after the beginning of the investigation. Although mortality is low, there is a significant increase in cases with cardiopulmonary involvement (40\% fatality). Thus, PAH screening in patients with AOSD should be considered and the prevalence of this association is likely to be underestimated in current studies. Early diagnosis and specific treatment can improve the survival of these patients. 